\title{
Outcomes Following Total Hip Arthroplasty: A Review of the Registry Data
}

\begin{abstract}
While total hip arthroplasty remains one of the most reliable procedures with excellent, cost-effective outcomes, there remains controversy in the choice of implant in terms of method of fixation, bearing surface, and size of the femoral head, especially in the younger population. This review looks at the possible information base that surgeons can explore before choosing the implant that they are comfortable with. It also looks at the findings of various registries, which readers can use in the process of informed consent. We have provided certain recommendations with specific reference to the method of fixation, bearing surface, and head size that can be backed by the available registry data. However, the information provided should be used only after considering local, financial, and patient-specific issues that surgeons encounter on a daily basis during their practice.
\end{abstract}

Keywords: Total hip arthroplasty, cemented total hip, uncemented total hip, joint registry data, National Institute for Health and Clinical Excellence

MeSH terms: Replacement, arthroplasty, hip, bone cements, review

\section{Introduction}

Total hip arthroplasty (THA) has revolutionized the quality of life of men and women of all ages since the 1960s, earning the title of "the operation of the century." In 2010, a survey of National Joint Registries (NJRs) estimated that around 959,000 annual primary and revision total hip procedures were being performed annually with the average rate at about 131 procedures per 100,000 population, and the average revision burden was found to be $12.9 \%$. Interestingly, $57.7 \%$ of the patients were women and $32.9 \%$ of patients were under the age of 65 years. $^{2}$ On the financial side, the global market for a hip replacement has been estimated to be around $\$ 4.8$ billion in 2014 with an estimated forecast of $\$ 5.9$ billion by $2020 .^{3}$

The clinical benefit and cost-effectiveness of the procedure are well proven. A systematic review of the cost-effectiveness has estimated the cost of a THA compared with no surgery at $\$ 10,402$ per quality-adjusted life year gained. Furthermore, if the World Health Organizations' suggested cost-effectiveness threshold of $<3$ times the gross domestic product, or $\$ 144,000$ based

This is an open access article distributed under the terms of the Creative Commons Attribution-NonCommercial-ShareAlike 3.0 License, which allows others to remix, tweak, and build upon the work non-commercially, as long as the author is credited and the new creations are licensed under the identical terms.

For reprints contact: reprints@medknow.com on 2011 data for the US, is used, then THA can be considered a highly cost-effective intervention. ${ }^{4}$

Long term survival of the prosthesis is well investigated in the general population, but it is also clear that the survival of implants is not as high in the young adults [Figure 1]. ${ }^{5}$

While the clinical benefits of a THR are well proven, there is a certain amount of confusion within the orthopedic community in relation to the myriad of prosthetic choices available for this procedure. Moreover, in an attempt to improve the outcome of an already proven prosthesis, especially in the younger population, surgeons have flirted with alternative bearing surfaces and methods of fixation.

Despite the vast amount of information available on this subject, it is difficult to get a unanimous view on the best type of prosthesis. The answers to these questions remain complex because of the large variety of the prosthesis available, clouded by variable data from various registries, published papers, and agenda of the industry. Furthermore, there are many factors to consider when choosing what prostheses to use for a particular

How to cite this article: Malviya A, Abdul N, Khanduja V. Outcomes following total hip arthroplasty: A review of the registry data. Indian $J$ Orthop 2017;51:405-13.

\section{Ajay Malviya, Nicole Abdul', Vikas Khanduja ${ }^{2}$}

Department of Trauma and Orthopaedics, Northumbria Healthcare NHS Foundation Trust, Wansbeck General Hospital, ' Specialist Trainee Year 4 in Trauma and Orthopaedics, Northern Deanery, Wansbeck General Hospital, Ashington, NE63 9JJ, ${ }^{2}$ Department of Trauma and Orthopaedics, Addenbrooke's Hospital, Cambridge, CB2 OQQ, UK

Address for correspondence: Mr. Ajay Malviya, Department of Trauma and Orthopaedics, Northumbria Healthcare NHS Foundation Trust, Wansbeck General Hospital, Ashington, NE63 9JJ, UK.

E-mail: ajay.malviya@ nhct.nhs.uk

Access this article online

Website: www.ijoonline.com DOI:

10.4103/ortho.IJOrtho_177_17 Quick Response Code:

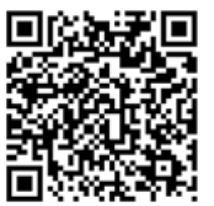



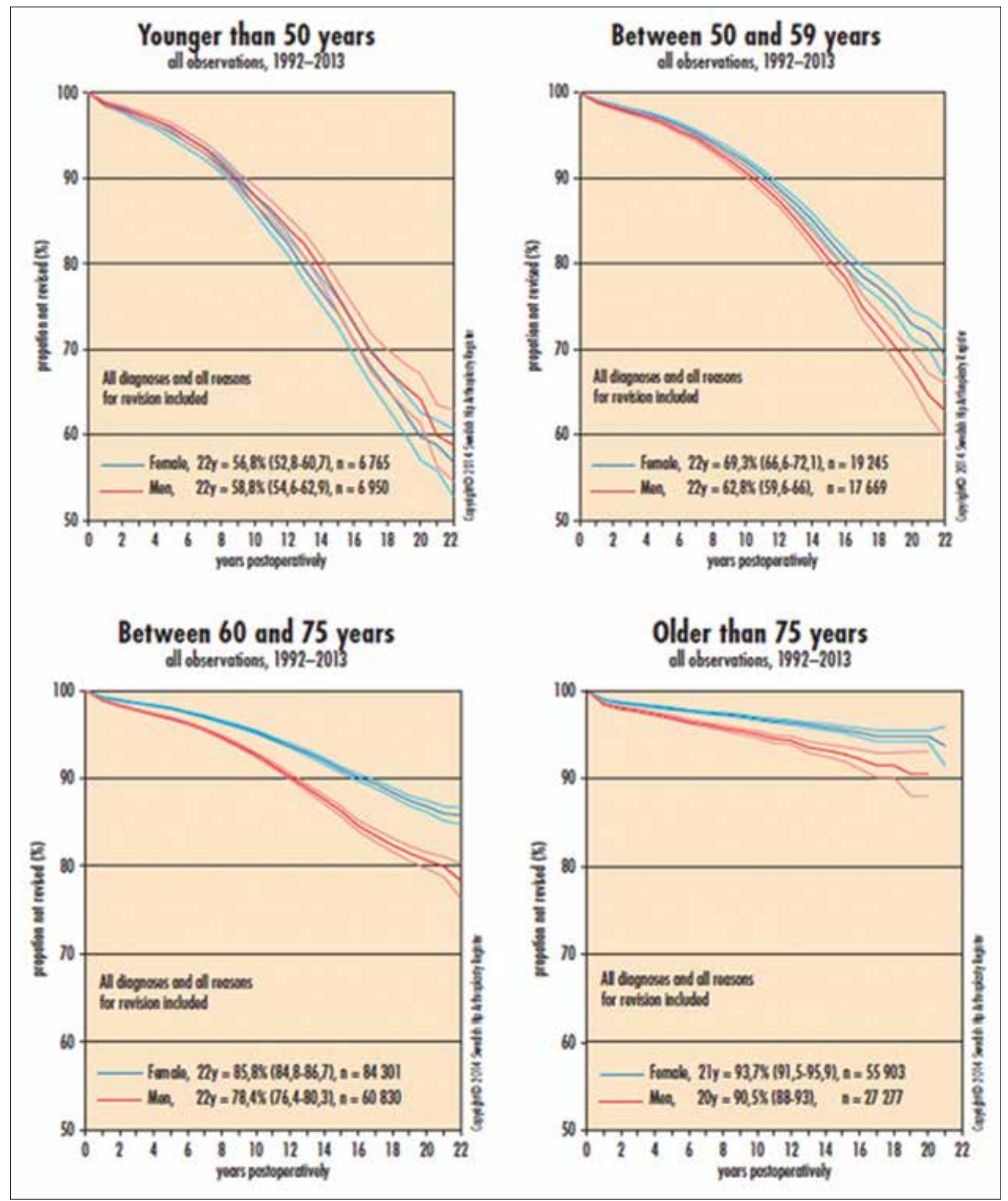

Figure 1: A graph showing the Swedish hip arthroplasty register data

patient including patients' demographics, the cost of the prosthesis, patient reported outcome measures and indeed training and comfort of using the implant.

The main aim of this review, therefore, is to discuss the controversies in the literature about the best method of fixation; the best bearing surface and the optimum size of the femoral head.

\section{Materials and Methods}

The review discusses in brief the various source of information that surgeons can reliably refer to in case of total hip arthroplasty surgery. The typical data available will be the revision rate and outcome measures from various registries and recommendation from bodies such as National Institute for Health and Clinical 
Excellence (NICE) and Orthopaedic Data Evaluation Panel (ODEP). Using revision data from registries may not be an accurate reflection because the indications for revision may vary between surgeons and there remain issues with regards to data capture. Moreover, the data reported in various registries have some degree of inconsistencies.

Following is the summary of our findings of the three controversial areas with a brief overview of bodies such as NICE, ODEP, and joint registries.

\section{National Institute for Health and Clinical Excellence}

NICE publishes guidelines for health professionals to aid decision-making and recommend treatments. Currently, NICE recommends only those hip prostheses which have a revision rate of $5 \%$ or less at 10 years. ${ }^{6}$ This guidance is for patients with end-stage arthritis and does not account for THRs done for other pathologies such as developmental dysplasia of the hip or Perthes disease. NICE also does not recommend a certain type of prosthesis, fixation method, or bearing surface over another. In their guidance, NICE also states that the average cost for a THR varies from $\leq 1557$ to $\leq 3869$, depending on the prosthesis and the materials used. ${ }^{6}$ Given the cost implications of THRs on a public funded system, it is important to determine specific recommendations for the type of prosthesis to be used in a specific subset of patients.

\section{Orthopaedic Data Evaluation Panel}

ODEP was set up in the UK in 2002 and independently evaluates hip prostheses against NICE guidance. ${ }^{7}$ ODEP assigns each implant a benchmark rating so that they can be compared with other prostheses on the market that meet the NICE guidance. Each implant given an ODEP rating is assigned a number $(10,7,5$, or 3$)$ and a letter (A or B). An implant assigned a 10 means that there is 10 years worth of data that is fully compliant with the NICE benchmarking. If an implant is assigned a 7, 5, or 3 it means there is 7 , 5 , or 3 years (respectively) of data, and the product is set to achieve the 10-year benchmark. The letter indicates the strength of the data; A meaning strong evidence and B meaning acceptable evidence. The final ratings that ODEP can assign are $3 \mathrm{~A}^{*}, 5 \mathrm{~A}^{*}, 7 \mathrm{~A}^{*}$, and $10 \mathrm{~A}^{*}$. If the implant has a revision rate of $<3 \%$ with a follow-up of at least 3 years, $3 \mathrm{~A}^{*}$ is awarded. If an implant has a revision rate of $<5 \%$ at 5,7 , or 10 years, $5 \mathrm{~A}^{*}, 7 \mathrm{~A}^{*}$, or $10 \mathrm{~A}^{*}$ is awarded, respectively, if the minimum number of years has been reached for each rating.

The use of ODEP rated implants as recorded in the NJR was determined in the $10^{\text {th }}$ annual report of the NJR for England, Wales and Northern Ireland. For implants with a $10 \mathrm{~A}$ rating on the NJR, a total of $88 \%$ of cemented stems, $69 \%$ of cementless stems, $34 \%$ of cemented acetabular components and only $3 \%$ of cementless acetabular components were used $^{8}$. These figures clearly show that although these products have been rated with a globally accepted system of benchmarking, autonomy still has a large part to play in choosing a prosthesis, along with patient-specific criteria and other available data on current implants.

The primary goal of conscientious surgeons would be to use a prosthetic combination and perform surgery that will meet the NICE benchmark of a $95 \%$ survival at 10 years, aided by the implants that have achieved the $10 \mathrm{~A}^{*}$ rating by ODEP. However, currently, there are 32 different acetabular components and 25 femoral stems that have achieved 10A* rating, again giving the surgeons a large variety of implants to choose from, but this remains a good database to explore before deciding on the final implant.

\section{Joint Registries}

Various NJRs have been set up over the last two to three decades.

\section{National Joint Registry}

The NJR for England, Wales, Northern Ireland, and the Isle of Man was established in 2002 and since then has collected data on joint replacements to monitor the performance and effectiveness of prostheses and surgery, respectively. Data from all joint replacements performed in the UK is submitted to the NJR, and the rate of compliance stands at $96 \%$ currently. ${ }^{8}$ It is the largest available registry with more than two million recorded procedures.

The Australian Orthopaedic Association National Joint Replacement Registry (AOANJRR)

This registry was set up by the Australian Orthopaedic Association in 1999. ${ }^{9}$ The registry has a large amount of data, with 59 different femoral and acetabular combinations coupled with 10-year outcome data. However, it is smaller than the NJR for England, Wales, Northern Ireland, and the Isle of Man, with data for approximately 453,950 hip replacements. Of this total, $15.7 \%$ are hip hemiarthroplasties, $72.5 \%$ primary THRs, and $11.8 \%$ revision THRs.

Unlike the NJR, the Australian Orthopaedic Association National Joint Replacement Registry divides THR into three different categories: total conventional arthroplasty, total resurfacing, and thrust plate. The third is not a method commonly used in the UK but includes an acetabular component and a femoral head prosthesis with a lateral fixation plate. However, conventional THR was the most popular choice in the registry, comprising $95 \%$ of all the data.

\section{Swedish Hip Arthroplasty Register}

This registry was first set up in 1979, making it the oldest registry in the International Society of Arthroplasty registers. ${ }^{5}$ The registry also contributes data to the Nordic Arthroplasty Register Association ${ }^{10}$ (NARA). However, the Swedish register dates back 28 years before the start of NARA. The most recent Swedish hip arthroplasty register annual report available is from 2014. Primary THR surgery 
gained popularity in Sweden between 1992 and 2013, increasing by nearly $80 \%$; in 2014 alone 16,565 THRs were carried out.

\section{Nordic Arthroplasty Register Association}

Another member of the International Society of Arthroplasty Registers is the NARA. ${ }^{10}$ This registry combines data from four Nordic countries: Denmark, Sweden, Finland and Norway. These four countries collaborated to form a common register in 2007 to try and improve the quality of joint replacement surgery. Before the countries collaborated, each of them had their own registry for a number of years; Denmark's registry was founded in 1995, Sweden's in 1979, Finland's in 1980, and Norway's in 1987. Due to the discrepancy of when each country set up a registry, the NARA includes hip arthroplasty data from 1997 up to the present day, but the most recent report published is from 2013. There are data for 620,261 primary hip replacements in the current report, the majority of these operations being performed in Sweden.

\section{Results and Discussion}

\section{Best method of fixation}

The 2015 NJR report ${ }^{8}$ shows that although uncemented implant use has declined since 2010, they are still the most used implants (39\%) of all THRs [Figure 2]. The use of hip resurfacing has declined over the past 6 years with an increase in the use of hybrid implants. Hybrid and reverse hybrid THRs accounted for $17.1 \%$ and $2.4 \%$, respectively, of all THRs.

The cemented THR revision rate $(3.63 \%$ 95\% confidence interval [CI] 3.43-3.83) was lower than the uncemented THR $(8.25 \%$ 95\% CI 7.90-8.62) at 11 years in the NJR. However, when this data are looked at closely, it seems that the higher revision rate was seen in metal-on-metal uncemented THRs as compared with ceramic-on-polyethylene uncemented THR, which had a revision rate of $3.62 \%(3.24-4.05)$. It should also be noted that the cemented ceramic-on-polyethylene THR revision rate was $2.98 \%$ and $2.15 \%$ for a hybrid THR.

The Swedish joint registry ${ }^{5}$ shows a similar trend, and although a cemented THR is the most common type of fixation, the popularity of it has decreased over recent years. Uncemented THR has increased instead. In 2014, $64.6 \%$ of all THRs were cemented, and $20.9 \%$ were uncemented. Results from 2014 data also show that the reverse hybrid THR dropped in popularity comprising only $11.2 \%$ of all operations, but hybrid THR gained popularity to $3 \%$ of all THRs. Age clearly has a bearing on the type of fixation [Figure 3], with the use of resurfacing or uncemented fixation being preferred in the younger population.

The Swedish joint registry has previously explored the survival of cemented and uncemented femoral and acetabular components. Interestingly, they found no significant difference between the acetabular component survival between the two although there was some survival benefit in the younger population with a reduced the risk of osteolysis and loosening up to 69 years of age [Figure 4], but this was associated with other problems that led to revision due to which the total revision risk showed no significant difference. The survival of the femoral component was significantly better for the uncemented components beyond 15 years [Figure 5]. However, during the first period after the operation, the risk of stem revision is greater if an uncemented stem is used; the reason for this difference is primarily revision for a periprosthetic fracture [Figure 5]. With the present selection of stem types, the risk was 8.8 (CI 6.41-

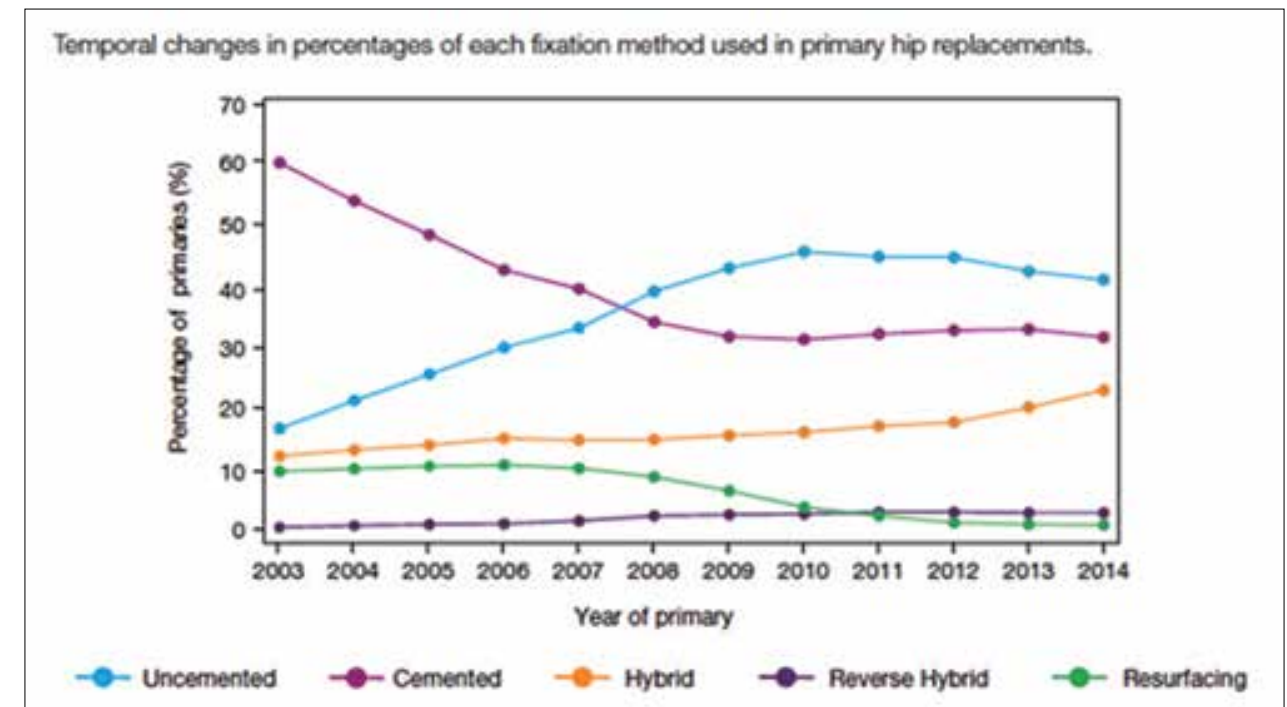

Figure 2: A graph showing National Joint Registry trend of prosthetic use 
12.04, $P<0.0001)$ times greater for revision due to to a periprosthetic fracture within 2 years if an uncemented stem instead of a cemented stem was used (Cox regression adjusted for age, gender and diagnosis). However, after about 8 years, there is a survival advantage of uncemented femoral components.

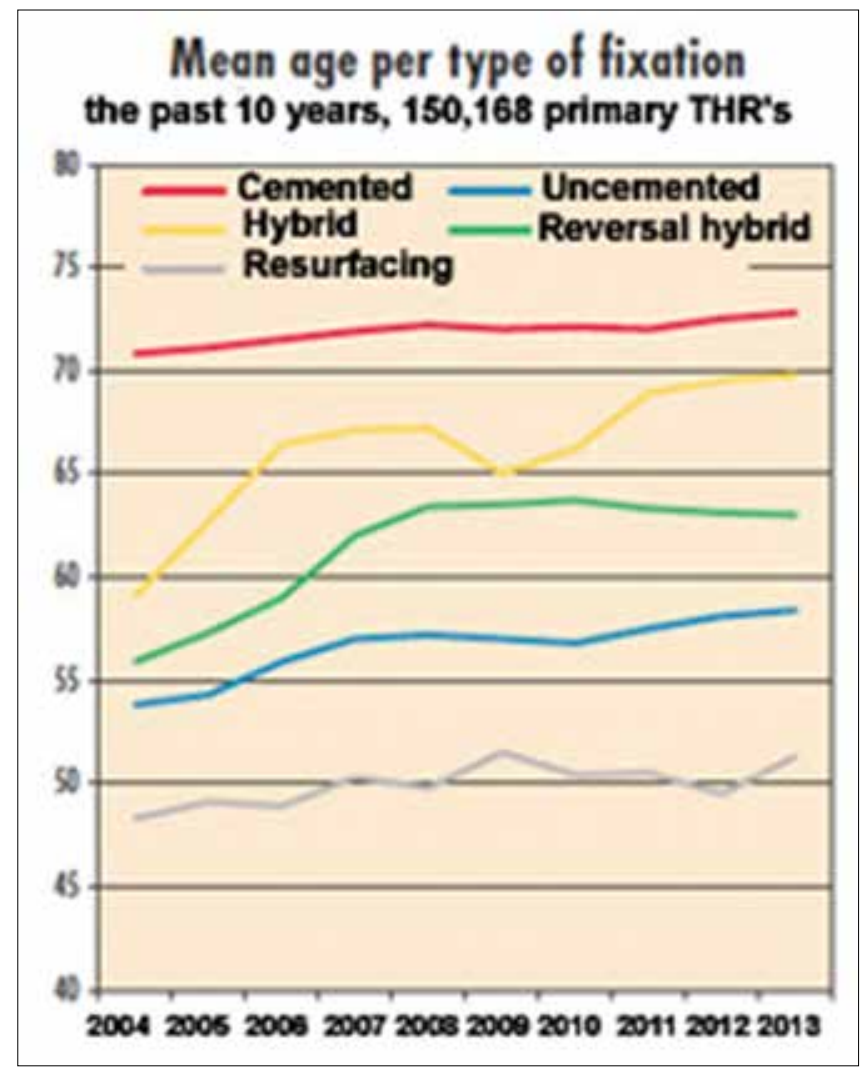

Figure 3: A graph showing the Swedish hip arthroplasty register - prosthetic use as per age
In the $\mathrm{AOANJRR}^{9}$ the trend of using cementless fixation has gained popularity, increasing to $63.2 \%$ in 2014 from $51.3 \%$ in 2003. This has led to a decline in the use of cemented fixation, dropping by $9.5 \%$ to $4.4 \%$ in 2014 and hybrid fixation dropping by $2.4 \%$ in the same timeframe. In 2014 , seven of the ten most popular femoral stems were uncemented; $67.3 \%$ of all conventional THRs were done using these ten most popular stems. Similarly, nine of the ten most popular acetabular components were uncemented, and $79.4 \%$ of all conventional THRs were done with these ten components. Furthermore, age and gender have been shown to affect revision rate. While men have a slightly higher overall revision rate, men and women over 75 years old have a lower revision rate as compared with other age groups after 6 months. It is also important to note that women have a lower revision rate the older they get, for men the relationship is not as clear. It was also noted that hybrid fixation had a lower revision rate compared with cemented or uncemented fixation. Interestingly again, in the first 3 months after surgery, uncemented THR had a higher revision rate compared with a cemented THR, but after 3 years, the opposite was true; this may well be related to a periprosthetic fracture. Overall, the data suggest that uncemented and hybrid fixation were better for the younger population ( $<75$ years), but for the over 75 , cemented combination performed more superiorly.

\section{Recommendation}

Most joint registry data favors the use of cemented prosthesis in the elderly population, the definition of which can be difficult, but physiological patients over the age of 65 years will do better with cemented prosthesis with prosthetic survival meeting the NICE benchmark of $>95 \%$ survival at 10 years. For the younger population, uncemented and
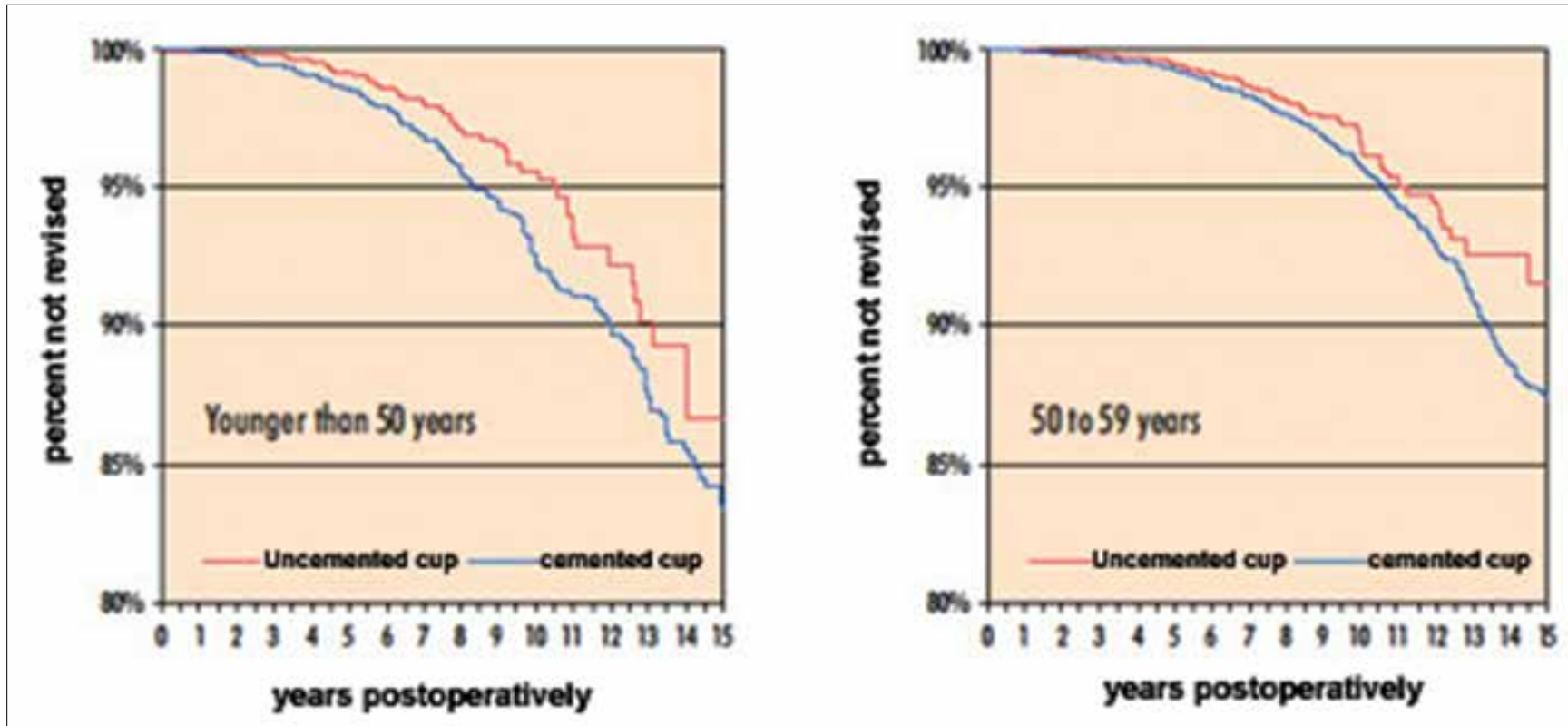

Figure 4: The Swedish hip arthroplasty register data comparing cemented and uncemented acetabular components in young patients 
hybrids are certainly good options to be considered to improve survival, but the results may not meet the NICE benchmark in patients younger than 50 years [Figure 1].

\section{Bearing surface}

In the $\mathrm{NJR}^{8}$ in 2014 , metal on polyethylene remained a popular choice for cemented $(84.4 \%)$, uncemented $(41.6 \%)$, hybrid (58.3\%) and reverse hybrid (64.4\%) THR, and is indeed the most common bearing surface throughout all the groups. Only a very small proportion of metal on metal bearing surfaces are used now $(0.1 \%)$. Of all the cemented THRs (36.1\%), metal-on-polyethylene was the most popular bearing surface used $(87.8 \%)$. This was true for uncemented
THR as well $(37.6 \%)$, but ceramic-on-ceramic was also a popular choice (34\%) [Figure 6]. The NJR data were very favorable for ceramic-on-polyethylene articulation regardless of whether they are cemented, uncemented or hybrids, so it is not surprising to see that these implants are becoming more popular. Ceramic on polyethylene seems to be the best bearing surface in all groups and when combined with hybrid hip replacement had the lowest combined revision rate of $2.15 \%(1.76-2.64)$.

In the AOANJRR ${ }^{9}$, the bearing surfaces used comprise ceramic, ceramicised metal, metal, cross-linked polyethylene, and noncross-linked polyethylene. The combination with the lowest revision rate at 14 years was

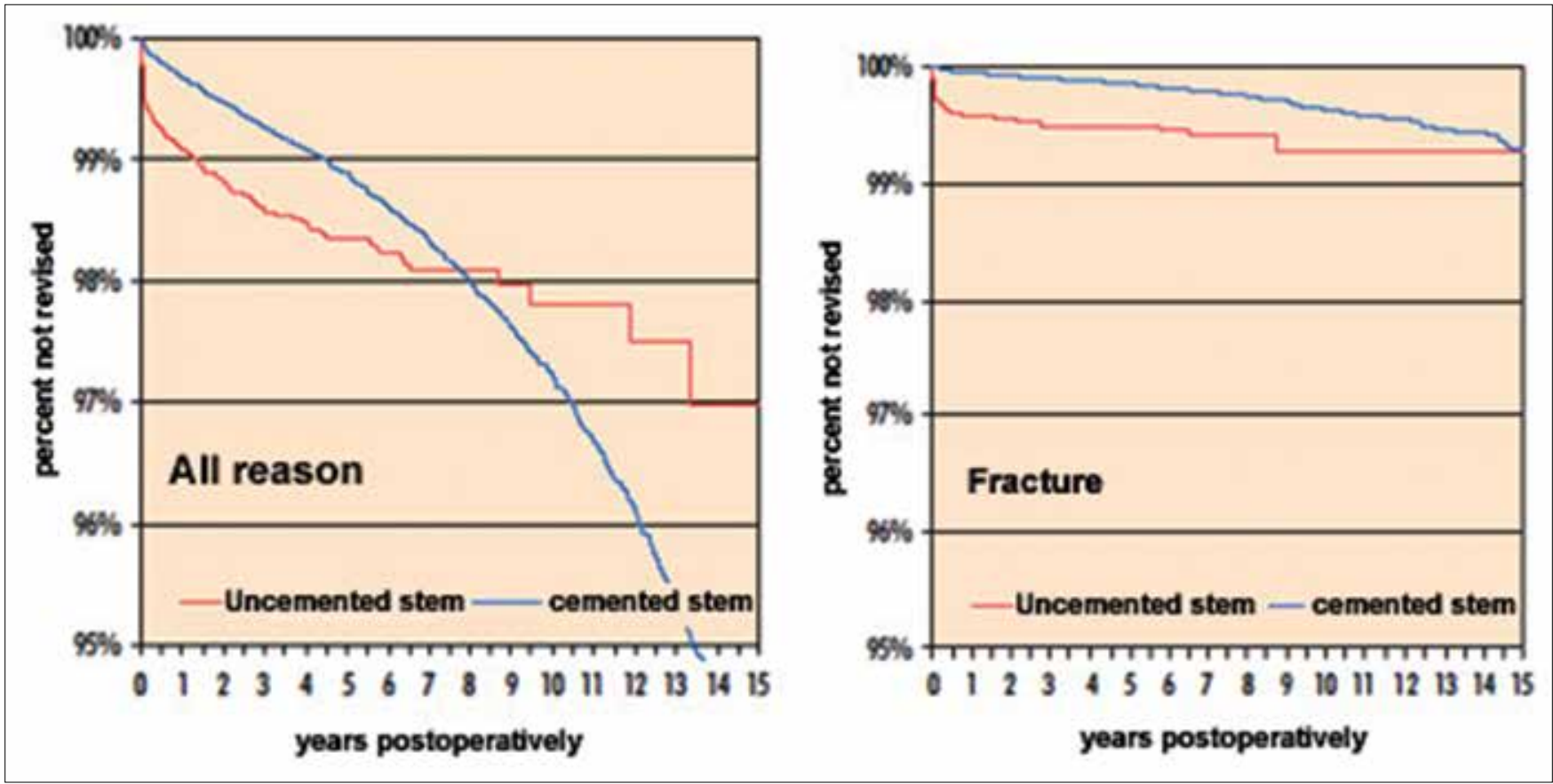

Figure 5: The Swedish hip arthroplasty register data comparing cemented and uncemented femoral components

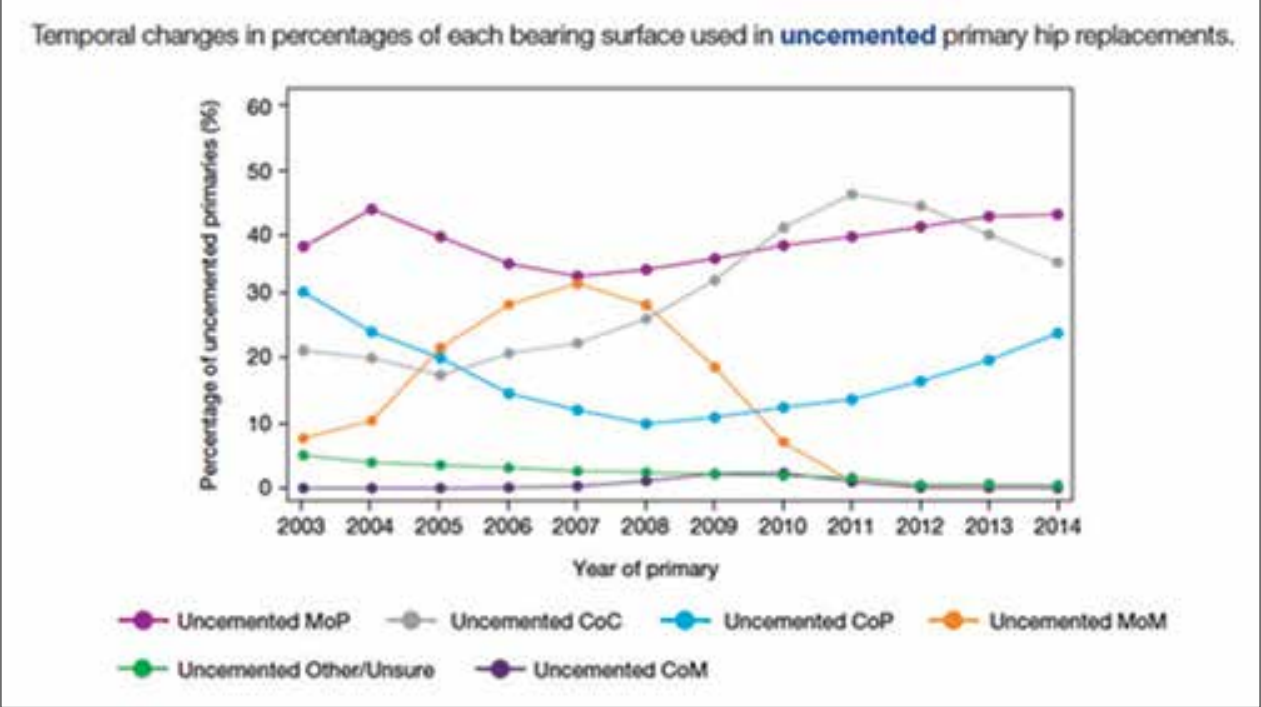

Figure 6: National Joint Registry - Temporal change in bearing surface used in primary hip replacement 
metal with cross-linked polyethylene (5.4\%); although, ceramic with cross-linked polyethylene also had low revision rates. The combination with the highest revision rate at 14 years was metal or ceramic femoral heads with noncross-linked polyethylene. Most ceramic on ceramic THRs had a cementless method of fixation (85.1\%), but they actually had a higher rate of revision compared with a hybrid method of fixation. Cross-linked polyethylene had a lower rate of revision compared with noncross-linked, regardless of whether metal, ceramic or ceramicised metal femoral head was used. In addition to the overall analysis of all the prostheses, the Registry had also undertaken analyses on five different acetabular components, each of which has been used in large numbers with both cross-linked and noncross-linked polyethylene. Three of the five prostheses had a lower rate of revision when cross-linked polyethylene was used. The other two did not show any difference.

In the Swedish registry ${ }^{5}$ metal with highly cross-linked polyethylene [Figure 7] remained the most popular bearing surface in the 2014 report (65.9\% THRs), but an increase in ceramic on highly cross-linked polyethylene $(10.9 \%)$ was also noted.

The use of highly cross-linked polyethylene is expected to reduce the risk for acetabular component/liner revisions after 5-12 years [Figure 8], the time in which osteolysis and/or loosening tends to result in a higher number of acetabular component revisions when using the older type of polyethylene.

\section{Recommendation}

While for the elderly population standard metal on polyethylene bearing surface will provide good results

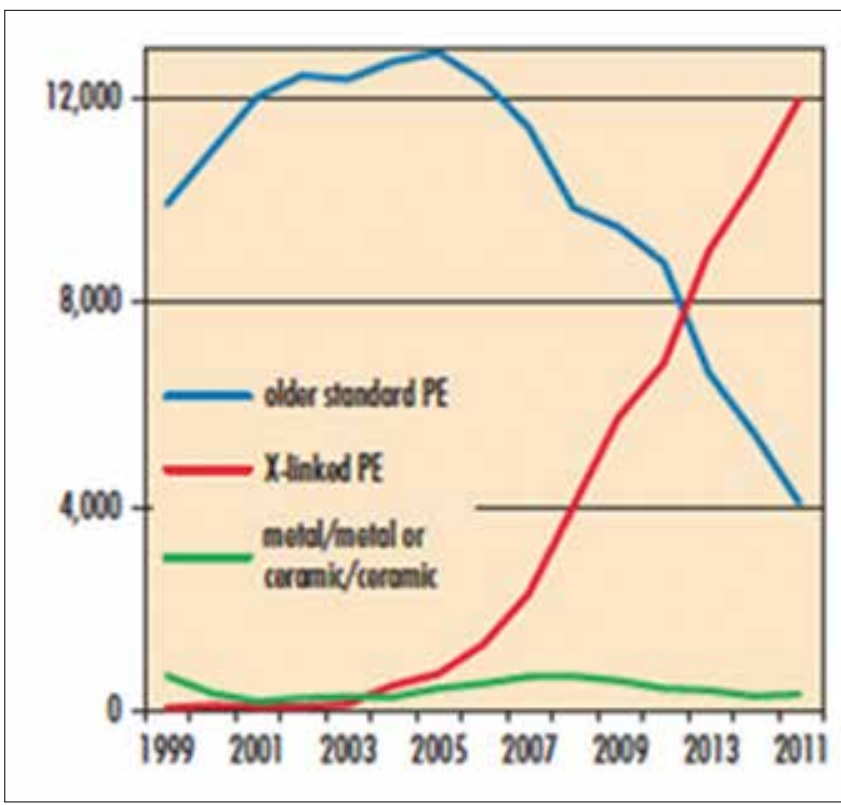

Figure 7: Use of cross-linked polyethylene- The Swedish hip arthroplasty register reaching the standards set by NICE $(>95 \%$ survival at 10 years), in the younger population there is benefit in using an alternative bearing surface with evidence leaning toward the use of ceramic on highly cross linked polyethylene, which would improve survival. The registry data are certainly more favorable towards this combination rather than the ceramic on ceramic articulation.

\section{Head size}

The usage of a larger head is gaining traction with the recent Swedish joint registry ${ }^{5}$ report showing that the most commonly used femoral head diameter was $32 \mathrm{~mm}(71.9 \%)$. The use of the $36 \mathrm{~mm}$ head had also increased by $0.8 \%$ to $10.2 \%$.

In the $\mathrm{NJR}^{8}$ for the metal on polyethylene articulation, the data are not conclusive but the best survival observed was for a head size of $<32 \mathrm{~mm}$ while for the ceramic on polyethylene articulation, the $32 \mathrm{~mm}$ head had the best survival. The rate of revision using a polyethylene monobloc acetabular component has been shown to be affected by head size. Using a ceramic or metal head of $36 \mathrm{~mm}$ with a cemented monobloc acetabular component had the highest rate of failure. On the uncemented side, a metal-on-polyethylene articulation with a head size of $44 \mathrm{~mm}$ had the worst outcome. In a ceramic-on-polyethylene articulation with an uncemented acetabular component, the $28 \mathrm{~mm}$ and $36 \mathrm{~mm}$ heads had the worst rates of failure.

In the AOANJRR ${ }^{9}$ head size affected rates of revision in a ceramic on ceramic THR. A $32 \mathrm{~mm}$ head size was found to have lower rates of revision when compared with a $28 \mathrm{~mm}$ head size or less, but there was no difference in

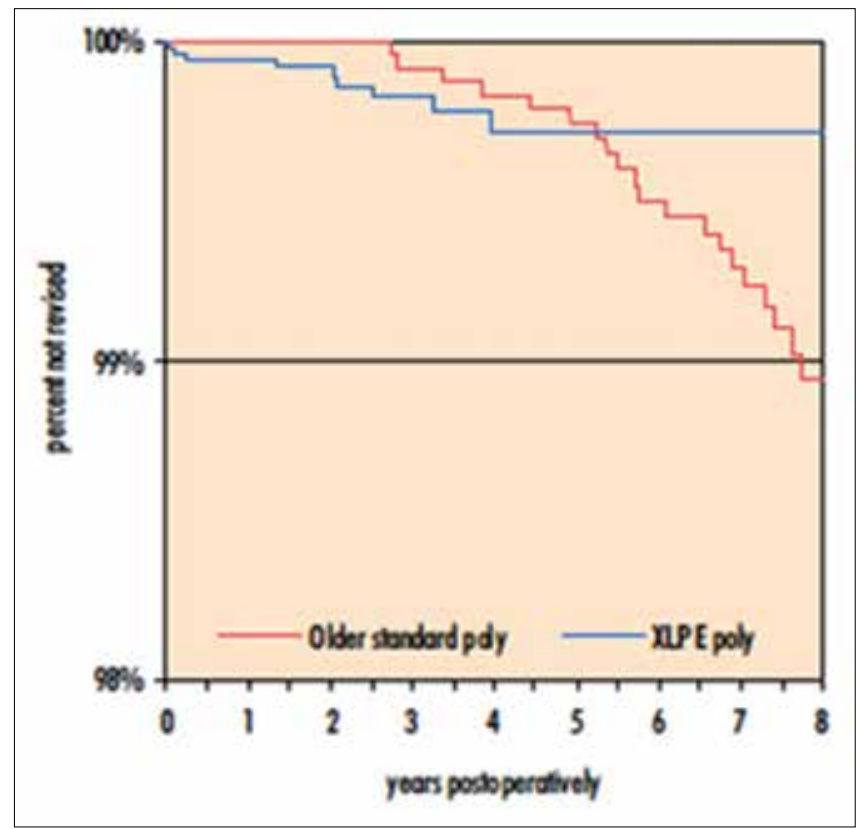

Figure 8: The Swedish hip arthroplasty register-Survival advantage of Highly crosslinked polyethylene 


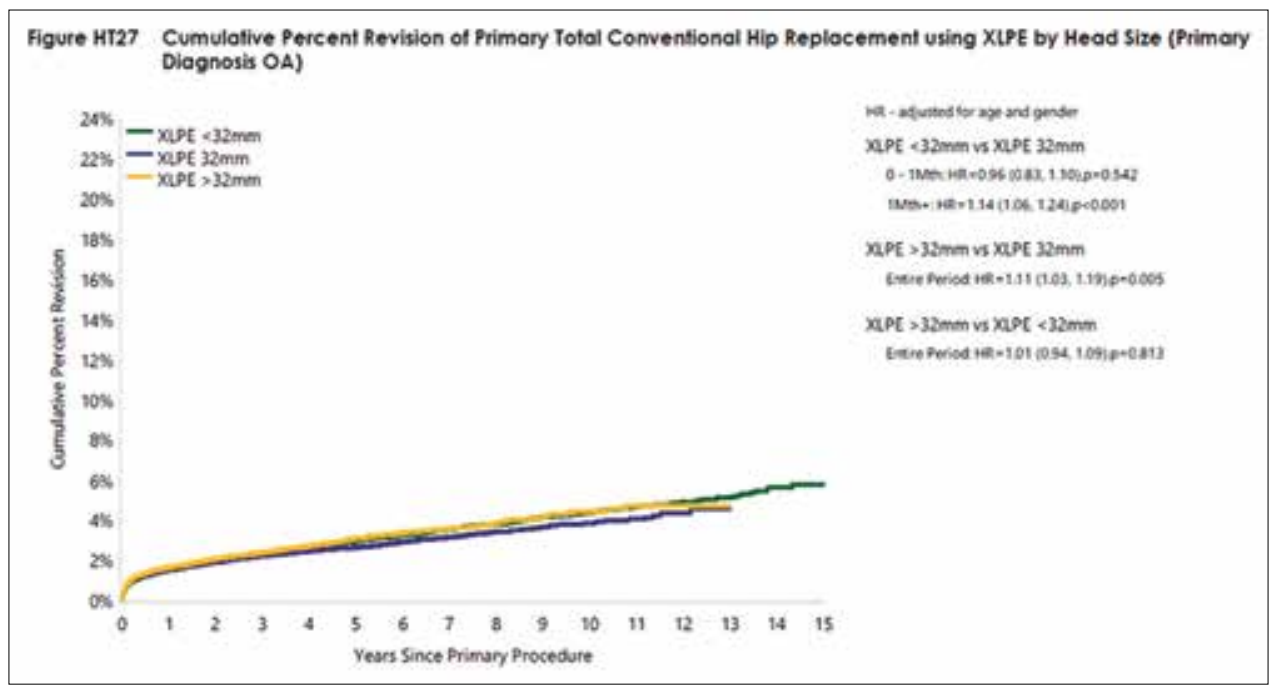

Figure 9: Australian Orthopaedic Association National Joint Registry - $32 \mathrm{~mm}$ head with highly crosslinked polyethylene is significantly better than with a head size more or less than $32 \mathrm{~mm}$

the rate of revision between a $32 \mathrm{~mm}$ and $36-38 \mathrm{~mm}$ head. A head size of $40 \mathrm{~mm}$ appeared to have a lower revision rate compared with a $32 \mathrm{~mm}$ head, but there was only a 5-year data set available for head sizes of $40 \mathrm{~mm}$ and above compared with 10 years for other head sizes. The AOANJRR data actually favors the use of a $32 \mathrm{~mm}$ head with cross-linked polyethylene [Figure 9].

\section{Recommendation}

For a standard metal on polyethylene articulation a head size of $32 \mathrm{~mm}$ or less will give better results, but for a ceramic on polyethylene articulation, the joint registry data are favorable toward the use of $32 \mathrm{~mm}$ head as compared with smaller or larger head sizes.

\section{Conclusion}

Using the data from the national registries, we can collate available information to potentially determine the best type of prostheses. In this review, we have explored the data in detail but have specifically excluded the brands of prosthesis because there is a significant regional variation in the availability of various prosthesis,

We also acknowledge that a number of factors need to be considered, including hip pathology, patients' demographics and cost among others while choosing a prosthesis. Although we have not gone into details of this, it is well known that cemented THRs are more cost effective than uncemented ones. ${ }^{11}$ Pennington et al. ${ }^{12}$ have shown that the cemented prostheses were the least costly type of THRs, but for most patient groups hybrid prostheses were the most cost effective. They concluded that uncemented prostheses did not provide sufficient improvement in health outcomes to justify their additional costs. Surprisingly, for such a common and important operation, the accurate costs and quality of life data are not readily available. However, with the ever-increasing amount of outcome data from the national databases available, the idea is that the choices can become more informed and we have attempted to do the same in this review.

All the registries have shown a decline in the use of a cemented THR in recent years, even though the cemented THR remains the most common implant used. Interestingly, Denmark and Sweden reported an equal usage of both cemented (50\%) and uncemented (50\%) THRs..$^{5,10}$

Although metal on polyethylene bearing surfaces is the most popular, there is promising data on ceramics as well, and certainly, for the younger adult, a ceramic on highly cross-linked polyethylene is the best way forward.

As far as the head size is concerned, a $32 \mathrm{~mm}$ or less comes up at the top for a metal head and a $32 \mathrm{~mm}$ for a ceramic head.

\section{Financial support and sponsorship}

Nil.

\section{Conflicts of interest}

There are no conflicts of interest. No benefits in any form have been or will be received by any of the authors as a result this publication.

\section{References}

1. Learmonth ID, Young C, Rorabeck C. The operation of the century: Total hip replacement. Lancet 2007;370:1508-19.

2. Kurtz SM, Roder C, Lau E, Ong K, Widmer M, Maravic $\mathrm{M}$ et al. International Survey of Primary and Revision Total Hip Replacement, in Presented at the $56^{\text {th }}$ Annual Meeting of the Orthopaedic Research Society, New Orleans; 2010.

3. Marketsandmarkets. HIP Replacement Market Worth 5.9 Billion USD by 2020 . Available from: http://www.marketsandmarkets. com/PressReleases/hip-reconstruction-devices.asp. [Last accessed on 2016 Jun 18]. 
4. Daigle ME, Weinstein AM, Katz JN, Losina E. The cost-effectiveness of total joint arthroplasty: A systematic review of published literature. Best Pract Res Clin Rheumatol 2012;26:649-58.

5. Garellick G, Karrholm J, Lindahl H, Malchau H, Rogmark C, Rolfson O. The Swedish Hip Arthroplasty Register Annual Report 2014 [Internet]. 2015. Available from: https://registercentrum. blob.core.windows.net/shpr/r/Annual-report-2014-BJv-q8pil. pdf. [Last Accessed on 2017 Jun 11].

6. NICE. Total hip replacement and resurfacing arthroplasty for end-stage arthritis of the hip [Internet]. 2014. Available from: http://www.nice.org.uk/guidance/TA304/chapter/1-Guidance. [Last accessed on 2017 Jun 11].

7. Tucker K. Industry Consultation on ODEP Ratings [Internet]. 2015. Available from: http://www.odep.org.uk/Portals/0/Images/ News_Articles/Industry_Consultation_On_ODEP_Ratings. pdf. [Last accessed on 2017 Jun 11].

8. The NJR Editiorial Board. National Joint Registry for England Wales, Northern Ireland and Isle of Man $12^{\text {th }}$ Annual Report 2015 [Internet]. 2015. Available from: http://www.njrreports.
org.uk/Portals/1/PDFdownloads/NJR\%2012th\%20Annual\%20 Report\%202015.pdf. [Last accessed on 2017 Jun 11].

9. Australian Orthopaedic Association National Joint Replacement Registry. Annual Report 2015 [Internet]. Adelaide: AOA; 2015. Available from: https://aoanjrr.sahmri. com/documents/10180/217745/Hip\%20and\%20Knee $\% 20$ Arthroplasty. [Last accessed on 2017 Jun 11].

10. Nordic Arthroplasty Register Association (NARA) Report 2015 [Internet]. Nordic Arthroplasty Register Association; 2016. Available from: https://stratum.registercentrum. se/\#!page?id=1150. [Last accessed on 2017 Jun 11].

11. Clarke A, Pulikottil-Jacob R, Grove A, Freeman K, Mistry H, Tsertsvadze A et al. Ageing populations OP75 NICE hips: Hip replacement interventions for osteoarthritis in the UK - A clinical and cost-effectiveness analysis. J Epidemiol Community Health 2014;68 Suppl 1:A37-8.

12. Pennington $M$, Grieve R, Sekhon JS, Gregg P, Black N, van der Meulen JH. Cemented, cementless, and hybrid prostheses for total hip replacement: Cost effectiveness analysis. BMJ 2013;346:f1026. 